\title{
Depth profiling of orientation in laminates with local lockin thermography
}

\author{
by KARPEN W., WU D., STEEGMÜLLER R., and BUSSE G.*
}

\author{
* Institut für Kunststoffprüfung und Kunststoffkunde (IKP), Universität Stuttgart, \\ Pfaffenwaldring 32,D-70569 Stuttgart, Germany
}

\begin{abstract}
Fibre reinforced materials are inspected with thermal waves. We show how orientation fields of carbon fibres can be probed both along the surface and in depth at low modulation frequencies and in short time. This technique is applicable for remote analysis and prediction of material properties.
\end{abstract}

\section{Introduction}

Fibre reinforced polymers have a high specific strength along fibre direction. To obtain materials whose strength is orthotropic in a given plane one uses laminates which consist of unidirectional layers with alternating directions.

Nondestructive testing methods to be integrated into a quality management system should therefore be capable to detect delaminated areas and to monitor fibre orientation. In both cases one is interested in local information (to be presented finally as an image) with lateral and in-depth resolution.

Aindow et al [1] describe how thermography can be used to monitor fibre-induced local thermal anisotropy. As a point source they use the fine tip of a soldering iron. A theoretical description of this problem was given by Krapez [2 - 4]. Also it has been shown that remote deposition of modulated heat results in phase information revealing local fibre orientation [5]. In this photothermal analysis [6] of thermal wave propagation there is a depth range limitation given by modulation frequency [7]. This is an advantage in terms of depth profiling, but it makes the method slow if application-relevant depth range is required because a phase image is recorded point by point with this technique. Pulsed methods [8] using a thermography camera provide information in a short time, but the concentrated energy deposition results in thermal load which may not be suited for sensitive materials. Also it is evident that a short pulse generates a broad spectrum of thermal waves where only the low frequency components are used to look deep into the material while in that case the high frequency part of the spectrum just heats the sample surface.

It has been described previously how the advantage of low frequency thermal waves can be combined with thermography to provide multiplex thermal wave ("lockin-thermography") information in a short time [9-11]. This paper describes the application of the technique to monitor fibre orientation and also its depth dependence.

\section{Experimental arrangement}

The basic idea of lockin-thermography as compared to conventional photothermal detection is shown in fig. 1. The conventional technique generates a point-like temperature modulation on a sample pixel which is monitored by a focused infrared detector. The signal is fed into a lockin-amplifier measuring the phase and magnitude of local response to modulated thermal input. Imaging is made by analysing pixel after pixel. 
http://dx.doi.org/10.21611/qirt.1994.042

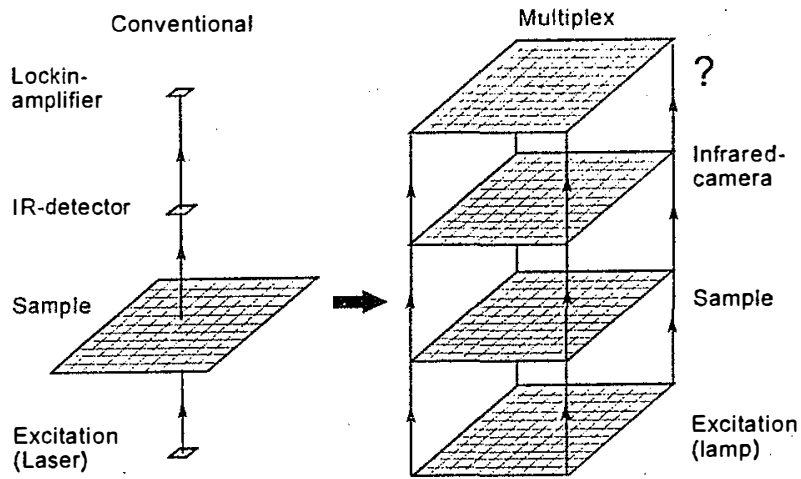

Fig. 1. - Conventional and multiplex photothermal imaging

It is quite obvious that it would be attractive to look at all pixels at the same time. In that case one generates the thermal wave at several points on the sample, and a thermography camera acts as an array of infrared detectors. However, to perform multiplex analysis based on photothermal detection each detector should be connected to its own lockin amplifier. With about $10^{5}$ pixels of a camera there is no hardware solution. It has been shown previously that for periodical thermal excitation which is coherent with the camera frequency one can reconstruct the thermal wave for each pixel and hence magnitude and phase [9]. If modulation frequency is one fourth of the camera frequency, one obtains for each cycle four thermographic "raw images" $S_{1}$ to $S_{4}$ from which the image of magnitude $A$ and of phase $\varphi$ is obtained according to

$$
A=\sqrt{\left(S_{3}-S_{1}\right)^{2}+\left(S_{4}-S_{2}\right)^{2}} \quad \text { and } \quad \varphi=\operatorname{arctg}\left(\left(S_{3}-S_{1}\right) /\left(S_{4}-S_{2}\right)\right) \text {. }
$$

From these equations it is obvious that $A$ is insensitive to superposition of unmodulated radiation, e.g. caused by reflection effects. As $\varphi$ contains only the ratio of differences its value is additionally independent of local power density or thermal emission coefficient. Therefore it is also independent of sample topography since the slope of contours affects only the noise of signal phase. This is true as long as edge-effects related to three dimensional heat flow can be ignored [12 - 13].



Fig. 2 - Multipoint excitation for fibre orientation imaging with lockin thermography 


\section{http://dx.doi.org/10.21611/qirt.1994.042}

The experimental setup used for multiplexed phase sensitive thermal wave ellipsometry is shown in fig. 2 where the beam of an Ar+-laser is split into an array of focal points. An acousto-optical modulator controlled by the synchronising computer provides sinusoidal modulation of illumination. Average power at each spot is typically $5 \mathrm{~mW}$. Around each spot we find a modulated temperature field which depends on fibre orientation.

\section{Results}

\subsection{Laminates}

To investigate the applicability of the method for orientation depth profiling a laminate sample was produced with two orientations $0 / 90$ where the thickness changed stepwise in such a way that the influence of the lower layer decreased from one edge of the sample to the other (fig. 3). The result obtained on this sample at $0.03 \mathrm{~Hz}$ with the arrangement of fig. 2 is also shown in fig. 3. Total time to obtain this image was 3 minutes including the evaluation of about 512 thermographic images. The elliptic shapes and the change of their excentricity is evident. It should be pointed out that the central spot corresponding to the zero order of grating diffraction looks different though it should not. The reason is that the higher power $(30 \mathrm{~mW})$ causes a higher temperature $\left(80^{\circ} \mathrm{C}\right)$ and therefore thermal diffusivity went up locally. This picture shows that overheating is a critical point for photothermal inspection of polymers.
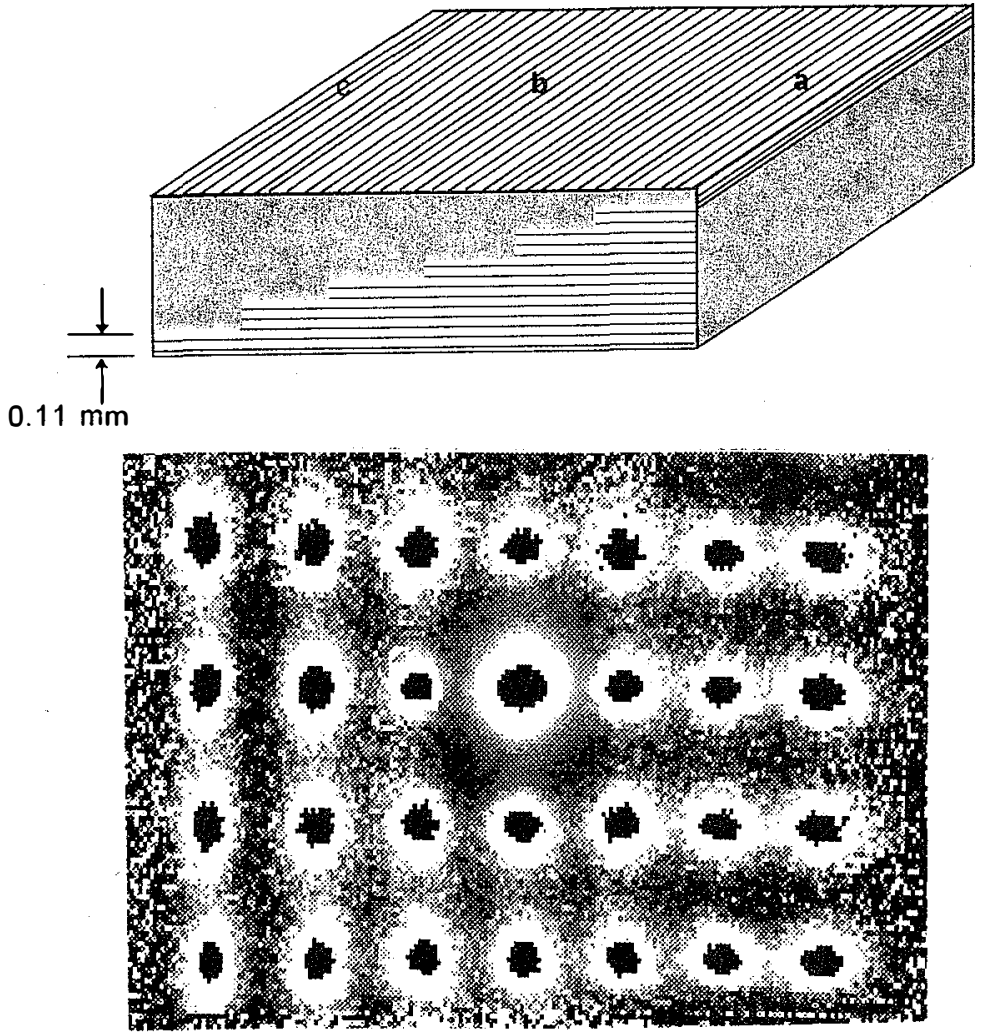

Fig. 3 - Multipoint lockin thermography: CFRP sample (top) and phase angle image (bottom) 
To investigate the change of elliptic patterns with more accuracy across the sample we performed also single spot measurements with a He-Ne laser beam of $15 \mathrm{~mW}$. It is evident that the inner part of the elliptical pattern responds more to the upper layer and the outer part to the lower layer. If one plots the ellipticity $a / b$ of the constant phase lines versus the average distance $(a+b) / 2$ of these lines from the laser spot one finds curves which characterise the thickness of the upper layer (fig. 4).

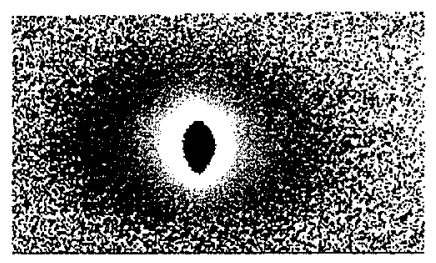

a

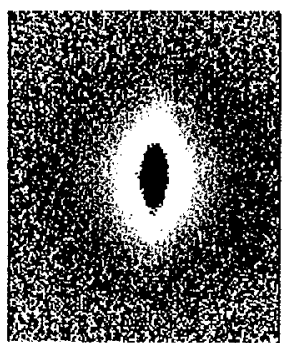

b

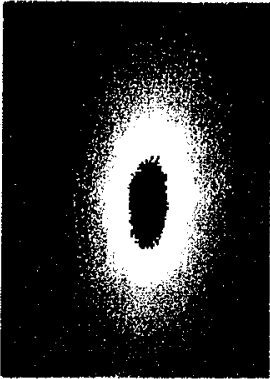

C

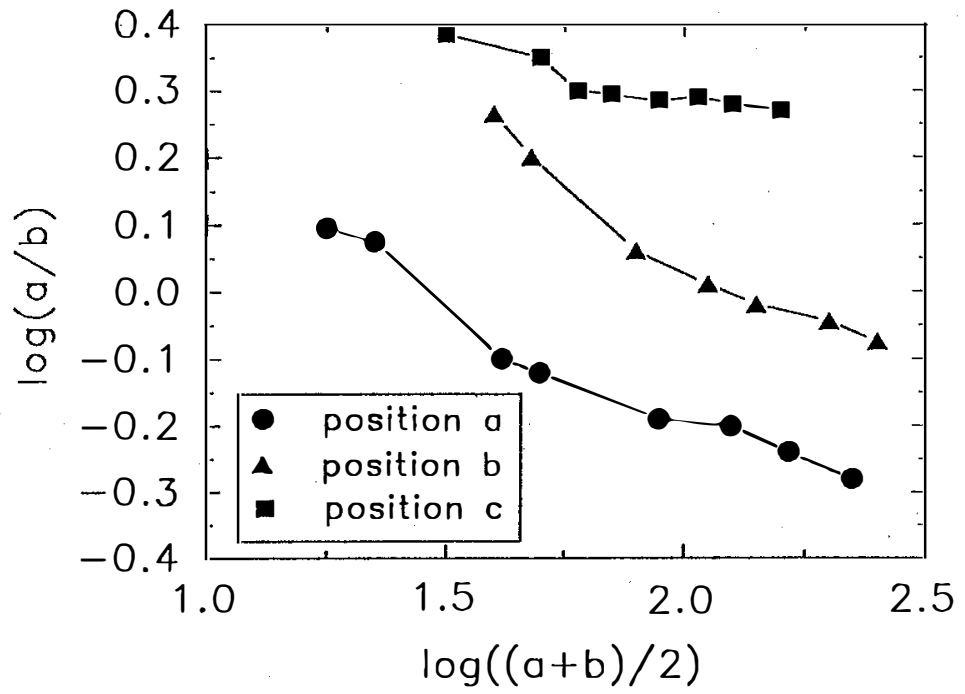

Fig. 4 - Ellipticity curves obtained from phase images $(0.03 \mathrm{~Hz})$ taken at positions a, b and c.

\subsection{Injection moulded polymers}

The advantage of phase angle imaging as compared to magnitude imaging or to conventional thermography is displayed in fig. 5 where part of the sample had been painted, this edge of paint was perpendicular to the fibres, and the laser spot was located near the edge. In fig. 5 the dark uncoated part is on the upper side resulting in higher infrared emissions. This way the elliptical shape is distorted for the left and middle 



Fig. 5 - Comparison of thermal ellipsometry (left) and phase sensitive thermal ellipsometry (magnitude: middle; phase: right) on a unidirectional CFRP sample partially covered with paint

images. An evaluation of them would provide wrong results. The phase image (right) displays the symmetrical pattern because the influence of the paint is suppressed.

M1

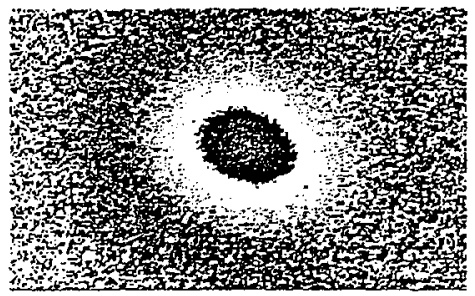

M2

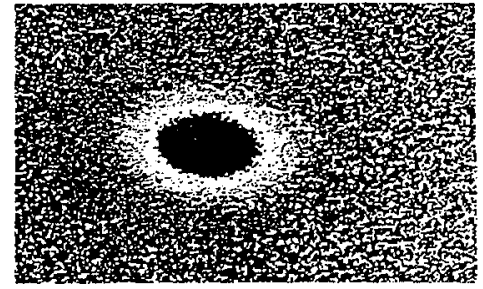

M3

Fig. 6 - Phase angle ellipsometry of injection moulded sample at locations M1 to M3

To produce components with complicated geometry one uses the injection moulding process. In that case fibre reinforcement can be achieved only with rather short fibres (typically $0.2 \mathrm{~mm}$ length). Due to the process with its flow gradients there is some torque acting on the fibres which results in a non-statistical distribution of orientation depending on the local flow characteristics. This partial alignment is of practical interest since it affects thermal extension and strength. Therefore we were interested to see how well thermal wave lockin ellipsometry is applicable to characterise fibre orientation in an 
injection moulded CFRP sample. The sample (fig. 6) has a hole in it. When the mould is injected from the left side it is split at the hole and meets in the seam area indicated by M2. Hence flow is oriented at M1 and M3 towards the seam. For these three focal point positions we could find phase fields (fig. 6) similar to the temperature fields induced in a contacting way some time ago [1]. In our case the increase of temperature generated remotely was about $55^{\circ} \mathrm{C}$. The observed orientation field is well consistent with results obtained with microwaves on glass fibre reinforced material [14].

\section{Conclusion}

We have shown that lockin thermal wave ellipsometry provides information on fibre orientation and on depth dependence of this orientation. The technique provides a fast nondestructive method to detect areas of local weakness and to eliminate faulty parts. This can significantly improve quality assurance of fibre reinforced polymers.

\section{Acknowledgement}

We are grateful to AGEMA for generous support of research on lockin thermography. Depth dependent fibre orientation is investigated in EC-project Nr. BRE2-CT92-0139.

\section{REFERENCES}

[1] AINDOW (J.D.), MARKHAM (M.F.), PUTTICK (K.E.), RIDER (J.G.), and RUDMAN (M.R.). -. Fibre orientation detection in injection-moulded carbon fibre reinforced components by thermography and ultrasonics. NDT INTERNATIONAL 19, 1986, p. 24.

[2] KRAPEZ (J.C.). - Analyse de la distribution superficielle de temperature produite par une source concentrée de chaleur à la surface d'un materiau composite formé de couches orthotropes. Application à la mesure de l'épaisseur de ces couches. Report RT91-010-121-02, IMI, Nat. Res. Counc. Canada, 1991.

[3] KRAPEZ (J.C.), CIELO (P.), MALDAGUE (X.), and UTRACKI (L.A.). - Optothermal analysis of polymer composites. Polymer composites 8, 1987, p. 396.

[4] KRAPEZ (J.C.) and CIELO (P.). - Transfert thermique dans un matériau formé de couches orthotropes. Eurotherm 4, June 1988, Nancy, France, p. 80.

[5] BUSSE (G.), RIEF (B.), and EYERER (P.). - Nondestructive evaluation of polymers with optically generated thermal waves. Polymer composites 8, 1987, p. 283.

[6] NORDAL (P.E.) and KANSTAD (S.O.). - Photothermal radiometry. Physica Scripta 20, 1979, p.659.

[7] ROSENCWAIG (A.). - Photoacoustic microscopy. American Lab. 11, 1979, p. 39.

[8] CIELO (P.), MALDAGUE (X.), DEOM (A.A.), and LEWAK (R.). - Materials evaluation 45, 1987, p. 452.

[9] BUSSE (G.), WU (D.), and KARPEN (W.). - Thermal wave imaging with phase sensitive modulated thermography. J. Appl. Phys. 71, 1992, p. 415.

[10] KUO (P.K.), FENG (Z.J.), AHMED (T.), FAVRO (L.D.), THOMAS (R.L.), and HARTIKAINEN (J.). - Parallel thermal wave imaging using a vector lock-in video technique. Photoacoustic and Photothermal Phenomena, (HESS (P.) and PELZL (J.), eds.): Springer ser. Topics in Optical Sciences 58, Springer, Berlin, 1987, p. 415.

[11] FAVRO (L.D.), AHMED (T.), JIN (H.J.), KUO (P.K.), and THOMAS (R.L.). - Noise suppression in IR thermal wave video images by real-time processing in synchronism with active stimulation of the target. SPIE Vol. 590,1985, p. 287.

[12] AAMODT (L.C.) and MURPHY (J.C.). - Effect of 3-D heat flow near edges in photothermal measurements. Appl. Opt. 21, 1982, p. 111.

[13] FRIEDRICH (K.) and WALTHER (H.G.). - The effect of edges on photothermal imaging. J. Appl. Phys. 70, 1991, p. 4697.

[14] HOLDEN (A.), ALLAN (P.S.), BEVIS (M.J.), DIENER (L.), BUSSE (G.) - Monitoring the multiple live-feed moulding process with microwave orientation imaging. German Plastics., 82, 1992, p. 28 